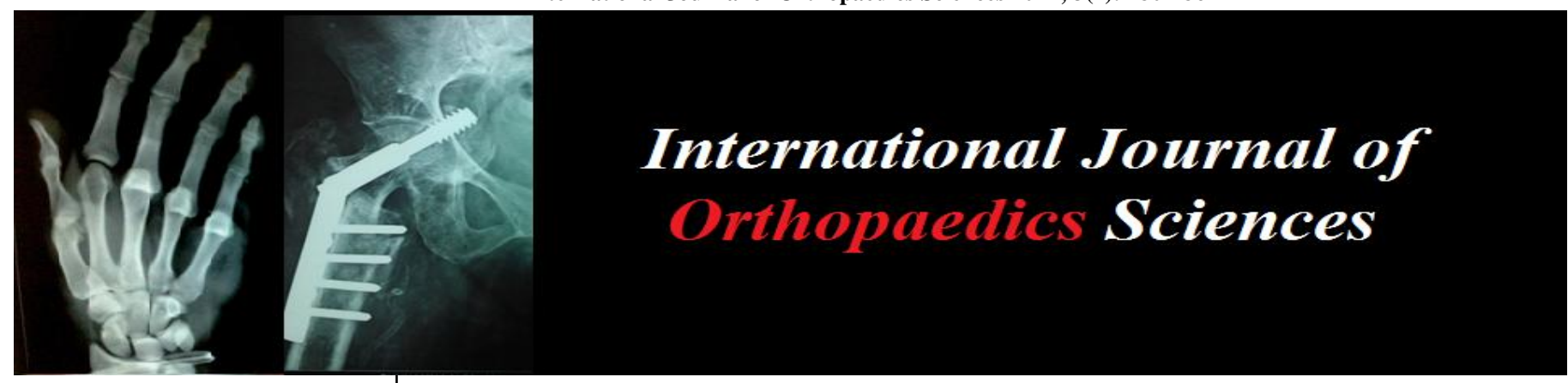

ISSN: $2395-1958$

IJOS 2017; 3(4): 780-786

(C) 2017 IJOS

www.orthopaper.com

Received: 19-08-2017

Accepted: 20-09-2017

Dr. Vishwanath C

Department of Orthopaedics, Adichunchangiri Institute of Medical Sciences, B G Nagar, Mandya, Karnataka, India

Dr. Sushanth B Mummigatti Orthopaedic Specialist, Aster CMI Hospital, Airport road, Bengaluru, Karnataka, India
Correspondence

Dr. Vishwanath C

Department of Orthopaedics, Adichunchangiri Institute of Medical Sciences, B G Nagar, Mandya, Karnataka, India

\section{Surgical outcome of tibial plateau fracture using minimally invasive technique with locking compression plate}

\author{
Dr. Vishwanath C and Dr. Sushanth B Mummigatti
}

DOI: https://doi.org/10.22271/ortho.2017.v3.i4k.109

\begin{abstract}
Introduction: The following study was conducted to examine the short term clinical and radiological results particularly early complications and healing rate of tibial plateau fractures treated with LCP by minimally invasive technique.

Methodology: The study was conducted in patients treated for tibial plateau fractures (type 1 to 4 Schatzker classification) at Adhichunchangiri Institute of Medical Science, BG Nagar from the month of May 2011 to May 2016. Fifty tibial plateau fracture patients were taken into the study, all were fixed with LCP using minimally invasive technique. Patients' age ranged from 20 to 70 years with a mean of 45 .

Results: The sample consisted of twenty patients with 38 males and 12 were females. The patients' ages ranged from 20-70 years with a mean age of 45 years. The causes of fractures were motorvehicle accident in 36 patients and fall in 14 patients. There were no sports or industrial accidents. 31 fractures involved the right side and 19 involved the left. The average length of hospitalisation was 12 days with a range of 7 to 20 days. The average number of days from injury to surgery was 5 days with a range of 2 to 10 days. The operative time ranged from 45 minutes to 100 minutes. Patients were followed up from 01 to 24 months. Functional outcome was rated as per NEER'S RATING SCORE, we got excellent results in 24 cases, good in 15, fair in 08 and poor in 03 patients.

Conclusion: Treatment of tibial plateau fractures using LCP provides good results. Since we have used minimally invasive technique soft tissue damage is minimal thus promoting good healing of fracture and less infection rates. Our early results were encouraging but long term studies are needed to prove definitively acceptable outcomes so that the technique can become part of the in the armamentarium of the orthopaedic trauma surgeon.
\end{abstract}

Keywords: Tibial plateau fracture, Schatzker classification, Minimally invasive technique, Locking Compression Plate, NEER'S Rating Score

\section{Introduction}

Tibial plateau fractures are on a raise due to increased incidence of road traffic accidents and also at the same time the surgical management for the same are also being modified continuously ${ }^{[1]}$. The luxuries of our life in the present time is at the cost of rapidly increasing industrialization, urbanization and mechanisation - so also the traumatic cases are on increase. Crowded cities, irregular traffic arrangement, fast moving vehicles are the most important contributory factors causing bony injuries, particularly polytrauma, comminuted fractures and also the soft tissue injury ${ }^{[2]}$. Tibial plateau fracture is one of them.

Tibial plateau fractures have been studied and reported extensively and exhaustively but still controversy exists over its management, whether surgical or conservative. Even undisplaced tibial plateau fractures should be operated, so that early mobilization of knee is possible ${ }^{[1]}$.

Before deciding the line of treatment one must bear in mind the following facts:

1. Extent of damage in tibial plateau fracture is often greater than what is seen on x-ray.

2. Malunion is exteremely common where as non-union is uncommon.

3. Painful knee and stiffness are the most serious and common complications of the tibial plateau fracture.

The LCP is an example of new technology which combines the principle of limited contact compression and locked internal fixation ${ }^{[3]}$. 


\section{Surgical and applied anatomy}

Fracture patterns in the proximal tibia are dictated by the forces applied combined with the osseous anatomy of the proximal tibia ${ }^{[2]}$. Occasionally, muscle forces or ligament attachments play a part in the fracture pattern. The tibia gradually flares from the relatively narrow diaphysis to the proximal tibia. In the proximal quarter, the anterior proximal tibia widens to become the tibial tubercle for attachment of the patellar tendon. Just above this, the proximal lateral tibia abruptly flares from the smooth anterolateral surface to form the lateral tibial condyle, which serves as the origin of the anterior compartment muscles, and more proximally has Gerdy's tubercle for the insertion of the iliotibial band ${ }^{[2]}$. Posteriorly on the lateral side, the fibular head serves as a palpable landmark and as the site of attachment of the fibular collateral ligament and the biceps tendon. The proximal tibiofibular joint is a synovial joint that may communicate with the knee joint.

On the medial side, the flare to the medial tibial condyle is more gradual directly medial and more abrupt and flared posteromedial. Angular forces to the knee and compression and axial loading lead to failure through these flared condyles on the lateral or medial sides or with straight axial loading on both sides. The medial plateau is more resistant to failure than the lateral plateau ${ }^{[4]}$.

The articular surface of the lateral tibial plateau is flat or slightly convex in relation to the medial tibial plateau that is concave, which provides greater congruity with the medial femoral condyle than on the lateral side ${ }^{[4]}$. The lateral plateau is also higher than the medial plateau, accounting for the few degrees of varus of the tibial plateau in relation to the shaft. The proximal articular surface slopes in relation to the shaft from the front, which is high, to the back, which is low. Assessing alignment in comparison to the nonfractured side is prudent.

Both the medial and lateral articular surfaces are covered by hyaline cartilage and are partially covered by the fibrocartilaginous menisci, both of which are attached to their respective plateaus by the meniscotibial ligaments (coronary ligaments) ${ }^{[5]}$. There is greater meniscal coverage of the lateral plateau than the medial plateau. The intercondylar eminence and medial and lateral tibial spines, which are nonarticular, separate the two plateaus. They also serve as attachment for the ACL anterior to the medial spine and the posterior cruciate ligament (PCL) that extends down to the posterior surface of the proximal tibia ${ }^{[2]}$.

The proximal anterior two thirds of the tibia are largely subcutaneous. The posterior tibia is deep beneath the structures crossing the popliteal fossa, making direct surgical exposures in this area difficult. The anterior tibia is more accessible but particularly the medial surface is at risk for surgical incisions in high-energy fractures. The pes tendons, gracilis, sartorius, and semitendinosis insert on the anteromedial portion of the proximal tibia distal to the insertion of the patellar tendon on the tibial tubercle. Before the insertion, these tendons give off expansions to the fascia of the lower leg. The anterior compartment muscles, tibialis anterior and extensor digitorum longus, arise from the inferior surface of the lateral condyle of the tibia. The origin must be elevated to place an anterolateral tibial plate ${ }^{[4]}$.

The common peroneal nerve runs under the cover of the biceps femoris and is on the back of the neck of the fibula. It is at risk from direct lateral impact mechanisms and with high-energy fractures of the tibial plateau, particularly medial plateau fractures which produce varus alignment. The tibial nerve in the popliteal fossa is rarely injured and rarely part of surgical approaches for tibial plateau fractures ${ }^{[5]}$.

The popliteal artery, which is at risk in knee dislocations, is rarely injured with tibial plateau fractures. However, the trifurcation of the popliteal artery occurs in an area where plateau displacement is likely with certain fracture patterns and the anterior tibial artery is bound at the interosseous membrane and is at particular risk in shaft-dissociated patterns. Occult injury to the anterior tibial artery may account in part for the compartment syndromes frequently associated with these fracture patterns.

\section{Mechanics of knee joint}

The mechanical axis of the femur does not coincide with the anatomical axis since a line traversing the centre of the hip joint and the centre of the knee forms an angle of $6^{\circ}-9^{\circ}$ with the axis of the shaft of the femur because of the disparity between the lengths of the articular surfaces of the femoral condyles and the tibial condyles, two types of motion during flexion and extension are produced:

1. Ginglymus (hinge)

2. Trochoid (pivot joint) articulation.

3. The joint permits flexion and extension in the sagittal plane and some degree of internal and external rotation when the knee is flexed.

The complex flexion and extension motion is a combination of rocking and gliding ${ }^{[3]}$. The rocking motion is demonstrable in the first $20^{\circ}$ of flexion, after which the motion becomes predominantly of the gliding type.

The natural deflection outward of the tibia on the femur, at the knee joint produces greater weight bearing stresses on the lateral femoral condyle than the medial.

But because the medial condyle of the femur is prolonged further forwards than the lateral condyle, the vertical axis of rotation falls in a plane near the medial condyle ${ }^{[4]}$.

The ratio of rolling to gliding is not constant. The ratio is $1: 2$ in early flexion and about $1: 4$ by the end of flexion

$\checkmark$ Flexion and extension ranges from $0^{\circ}-140^{\circ}$.

$\checkmark$ Rotation ranges from $5^{\circ}-25^{\circ}$ with individual variation.

$\checkmark \quad 5-10^{\circ}$ of hyperextension is also possible.

$\checkmark$ Medial meniscus is more prone for injury because the anchorage of the medial meniscus permits less mobility.

\section{Screw-home movement}

The articular surface of the medial condyle is prolonged anteriorly, and as the knee comes into fully extended position, the femur internally rotates until the remaining articular surface on the medial condyle is in contact ${ }^{[5]}$. The posterior portion of the lateral condyle rotates forward laterally, thus providing a screwing home movement, locking the knee in the fully extended position

When flexion is initiated unscrewing of the joint occurs by external rotation of the femur on the tibia.

$\checkmark$ Normal sagittal excursion of tibia on the femur not more than $3-5 \mathrm{~mm}$.

$\checkmark$ Normal varus and valgus motion at the knee, when extended not more than $6^{\circ}-8^{\circ}$.

\section{Mechanism of injury}

Tibial plateau fractures are intra-articular fractures of the proximal tibia that occur in adults, with the highest incidence in the third to fifth decades of life ${ }^{[5]}$. Fractures in men occur at a younger age and women have increasing incidence with advancing age, particularly in the sixth and seventh decades, which indicates these fractures are occurring in osteopenic bone ${ }^{[5]}$. 
Diverse patterns of tibial plateau fractures result when the knee sustains one of a variety of mechanisms of injury. In middle-aged or elderly patients, simple falls lead most commonly to lateral or, less commonly, medial side fracture patterns. Split depression fractures of the lateral plateau are most common. When the bone is very osteopenic, insufficiency fractures in elderly patients can occur and be missed on plain radiographs. Higher-speed injuries in younger patients from sports or similar mechanism can cause split fractures or rim avulsion fractures associated with knee ligament injuries. Motor vehicle accidents and falls from heights and pedestrian struck injuries often produce more severe patterns, which may involve both condyles and have a high risk for associated neurovascular injuries, compartment syndrome, and communicating open wounds ${ }^{[5]}$.

The magnitude, type, and direction of forces that injure the knee dictate the fracture pattern. The greater the energy absorbed by the proximal tibia, the more severe is the fracture and the more the fragments are displaced and comminuted. The energy of fracture results from a combination of the forces applied and the quality of the bone [5]. Generally, axially loading forces are more rapid and release greater energy than angular forces. In cadavers, it is possible to produce typical split fractures with pure valgus forces, local compression fractures with axial forces, and split depression fractures with combinations of both forces.

The proximal tibia is most likely to be subjected to a valgus force because of the normal 5 to 7 degrees of valgus alignment of the knee and because of a propensity to be struck from the lateral side ${ }^{[2]}$. A valgus force loads the lateral tibial plateau to failure from direct impact with the lateral femoral condyle. A combination of valgus and axial compression produces lateral side depression, split depression, or, less commonly, lateral split or total lateral condyle fractures. Younger patients with good bone tend to have split fractures with less depression, and elderly patients with osteopenic bone have a greater component of compression with a less prominent split fragment. Most commonly in lateral fracture patterns, there is at least a small component of both a split fracture and depression at the peripheral margin of the fracture. Less commonly than in lateral side fractures, varus injuries lead to failure of the medial plateau. These injuries can involve the entire medial plateau and, in some cases, the fracture-shearing plane may extend well into the lateral plateau.

Tibial plateau fractures most often occur with the leg in a weight-bearing position so axial load is typically some component of the injuring force ${ }^{[2]}$. Generally, the greater the axial load component, the more energy there is at failure and the more severe is the fracture pattern. Bicondylar patterns result when axial load predominates, with the severity varying based on the magnitude of the axial forces. Occasionally in a patient with a valgus knee, an axial force may shear the medial tibial condyle and produce a medial plateau fracture or fracture dislocation.

Another type of tibial plateau fracture pattern occurs at the metaphyseal region as a result of direct trauma and/or a combination of axial load and bending forces ${ }^{[4]}$. These are classic bumper injuries or other crush, direct blow, or similar mechanisms where the tibial shaft is separated from the condyles with proximal extensions of fracture lines into the plateau. These severe injuries have a high risk of complications because of both the area of the injury and the high degree of energy transfer ${ }^{[5]}$.

\section{Pathology of fractures of tibial plateau}

Pure split fractures are commoner in young patients ${ }^{[7]}$.

1. Depressed fractures and split depressed fractures are common in older patients.

2. The fracture line and degree of flexion of knee combined with valgus/varus strain and axial loading contribute to determine the fracture line and the site of depression whether it is anterior, middle OR posterior.

3. Collateral ligament integrity and forces determine the type of fracture.

4. Pure axial loading or axial loading combined with varus/valgus stress determines the type of bicondylar fractures.

5. Violent injuries are associated with ligament injures, vascular and nerve injures.

6. Direct injuries to the upper tibia can also lead to subcondylar fractures without involving the articular surface.

\section{Materials and methods}

A Prospective Study on tribial plateau fracture fixed with locking compression plate using minimally invasive technique was conducted in the period from MAY 2011 to MAY 2016 at Adichunchungiri Institute of Medical Sciences.

Study population: All Male\Female patients aged 20 to 70 years admitted in the hospital.

Study sample size: Fifty patients who were admitted and operated between MAY 2011 to MAY 2016 and had fulfilled the inclusion criteria, were enrolled for this study.

\section{Study type: Prospective study.}

Study title: Surgical management of tibial plateau fracture using minimally invasive technique with locking compression plate.

Duration of study: May 2011 to May 2016.

The duration of follow up ranged from 6 months to 24 months. All the fractures in this series were post-traumatic. No pathological fracture was included in the study.

\section{Surgical procedure}

All surgeries were performed on an elective basis using standard aseptic precautions surgery was performed under spinal or General anaesthesia. Patient is placed supine on a radiolucent table with a pillow below the distal thigh, the entire injured extremity is prepared and draped. The ipsilateral iliac crest was prepared and draped for possible autogenous bone grafting. Perioperative intravenous antibiotics were administered. Fracture was reduced indirectly under fluoroscopic guidance a $2 \mathrm{~mm} \mathrm{k}$-wires used as a joystick to reduce and hold fracture fragments. Temporay fixation is done using k-wires. An incision of 2 to $3 \mathrm{~cm}$ over proximal aspect of tibia over the medial or lateral side (depending on fracture pattern) was taken. A submuscular plane was created and a precontoured stainless steel $4.5 \mathrm{~mm}$ locking compression plate was slid in the anterior submuscular plane. The plate was held in place by k- wires. It was then fixed with screws which were inserted percutaneously through the primary surgical incisions. Distally 3.2 drill bit was used for drilling and $4.5 \mathrm{~mm}$ Cortical screws were inserted and fixed. Reduction satisfactory. Wound closed. 
Results

Table 1: Age distribution

\begin{tabular}{|c|c|c|}
\hline Age (Years) & No. of patients & Percentage (\%) \\
\hline $20-30$ & 16 & 32 \\
\hline $31-40$ & 12 & 24 \\
\hline $41-50$ & 09 & 18 \\
\hline $51-60$ & 08 & 16 \\
\hline $61-70$ & 05 & 10 \\
\hline TOTAL & 50 & 100 \\
\hline
\end{tabular}

Table 2: Sex distribution

\begin{tabular}{|c|c|c|}
\hline Sex & No of patients & Percentage (\%) \\
\hline Male & 38 & 68 \\
\hline Female & 12 & 24 \\
\hline
\end{tabular}

Table 3: Mechanism of injury

\begin{tabular}{|c|c|c|}
\hline Mechanism of injury & No. Of case & Percentage (\%) \\
\hline Road traffic accident & 36 & 72 \\
\hline Fall from height & 14 & 28 \\
\hline Total & 50 & 100 \\
\hline
\end{tabular}

Table 4: Type of fracture

\begin{tabular}{|c|c|c|}
\hline Type & No. Of fracture & Percentage \\
\hline Open & 09 & 18 \\
\hline Closed & 41 & 82 \\
\hline
\end{tabular}

Table 5: Classification

\begin{tabular}{|c|c|c|}
\hline Schatzker & No of cases & Percentage \\
\hline Type 1 & 12 & 24 \\
\hline Type 2 & 28 & 56 \\
\hline Type 3 & 07 & 14 \\
\hline Type 4 & 13 & 26 \\
\hline Type 5 & 00 & 00 \\
\hline Type 6 & 00 & 00 \\
\hline
\end{tabular}

Table 6: Side distribution

\begin{tabular}{|c|c|c|}
\hline Side & No of patients & Percentage (\%) \\
\hline Right & 31 & 62 \\
\hline Left & 19 & 38 \\
\hline
\end{tabular}

Table 7: Operative time

\begin{tabular}{|c|c|c|}
\hline Operative time & No. of fracture & Percentage \\
\hline $0-45 \mathrm{~min}$ & 11 & 22 \\
\hline $45-90 \mathrm{~min}$ & 35 & 70 \\
\hline $90-135 \mathrm{~min}$ & 04 & 20 \\
\hline
\end{tabular}

Table 8: Time to union

\begin{tabular}{|c|c|c|}
\hline Union (weeks) & No. of cases & Percentage \\
\hline$<14$ & 10 & 20 \\
\hline $14-16$ & 17 & 34 \\
\hline $16-18$ & 16 & 32 \\
\hline $18-20$ & 07 & 14 \\
\hline
\end{tabular}

Table 9: Full weight bearing achieved

\begin{tabular}{|c|c|c|}
\hline Achieved times (weeks) & No. of cases & Percentage \\
\hline$<16$ & 08 & 16 \\
\hline $16-18$ & 18 & 36 \\
\hline $18-20$ & 15 & 30 \\
\hline $20-22$ & 09 & 18 \\
\hline
\end{tabular}

Table 10: knee flexion

\begin{tabular}{|c|c|c|}
\hline Knee flexion (degrees) & No. of cases & Percentage \\
\hline$<90$ & 07 & 14 \\
\hline $91-119$ & 19 & 38 \\
\hline$>120$ & 24 & 48 \\
\hline
\end{tabular}

Table 11: Complications

\begin{tabular}{|c|c|c|}
\hline Complication & No. of cases & Percentage \\
\hline Stiffness & 12 & 24 \\
\hline Delayed union & 05 & 10 \\
\hline Infection & 03 & 06 \\
\hline Knee pain & 07 & 14 \\
\hline Skin necrosis & 00 & 00 \\
\hline Implant failure & 00 & 00 \\
\hline Implant exposure & 00 & 00 \\
\hline
\end{tabular}

Table 12: Functional rating as per neer's rating score

\begin{tabular}{|c|c|c|}
\hline Rating (in points) & No. of cases & Percentage \\
\hline Excellent (more than 85) & 24 & 48 \\
\hline Good (70-85) & 15 & 30 \\
\hline Fair (55-69) & 08 & 16 \\
\hline Poor (less than 55) & 03 & 06 \\
\hline
\end{tabular}

Case Series: Case 1

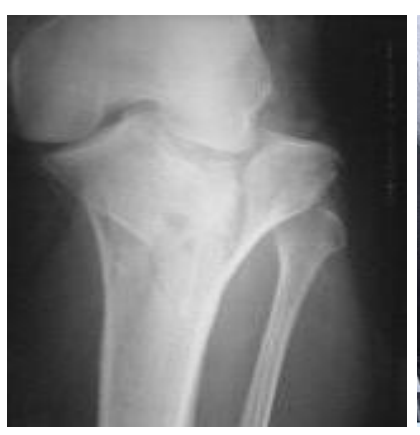

PRE OP Xray

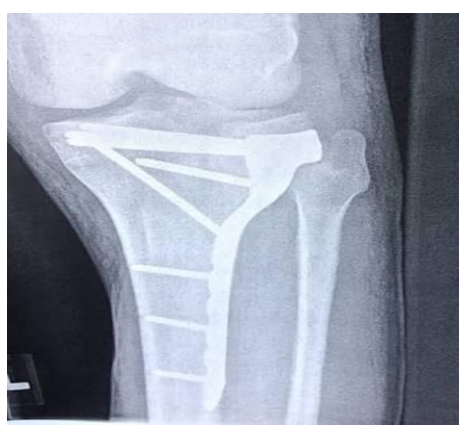

Post OP Xray

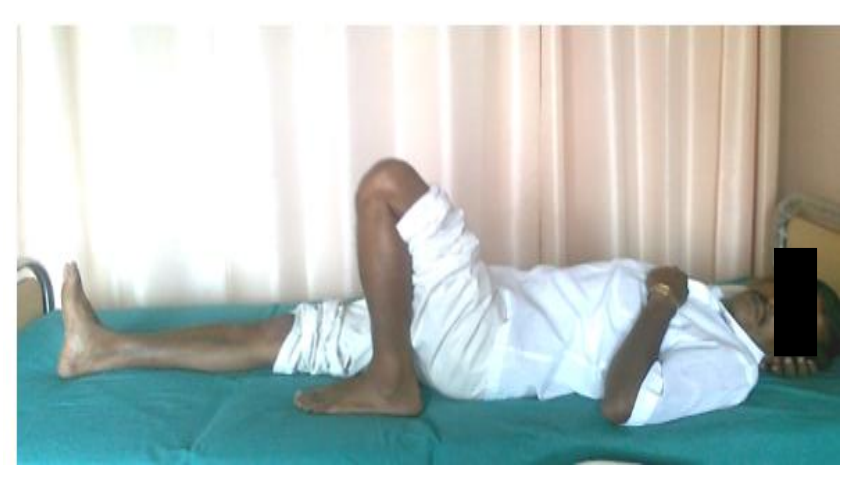

Flexion

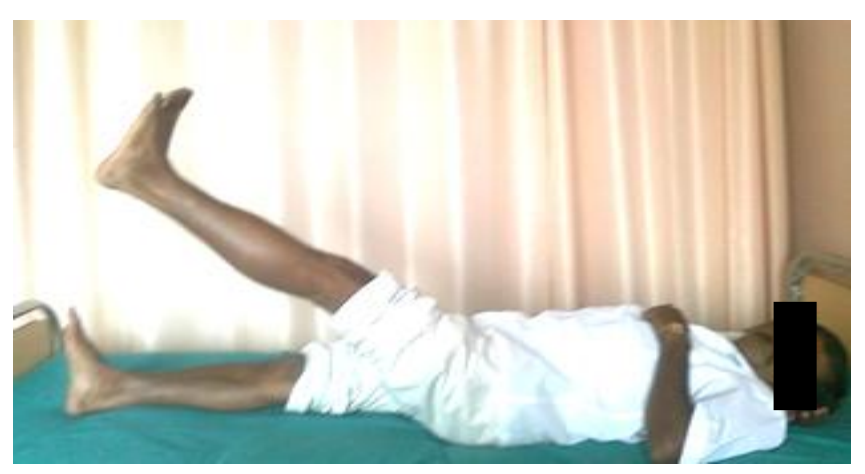

Extension 


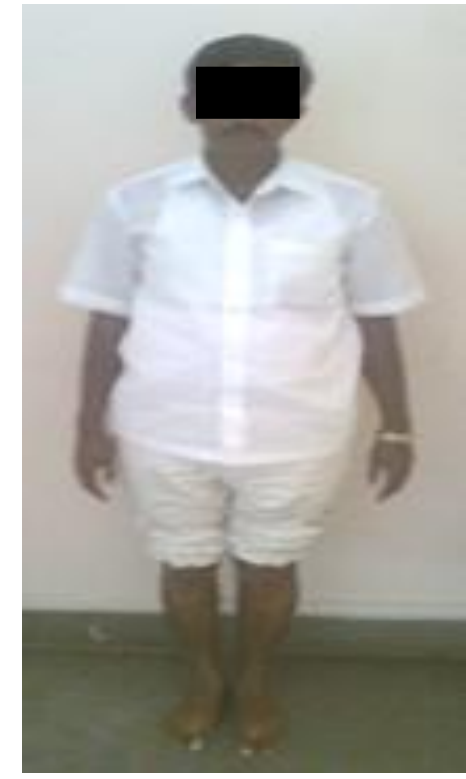

Full Weight Bearing

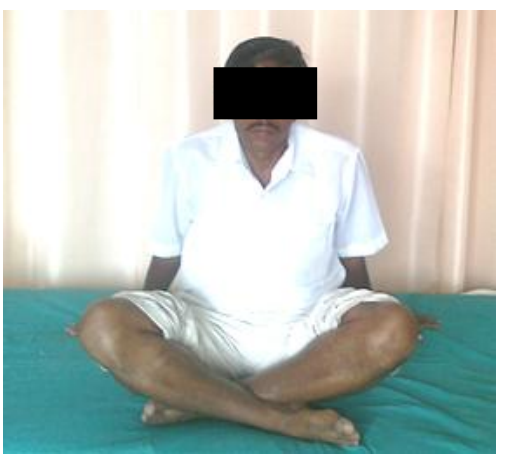

Sitting Crosslegged

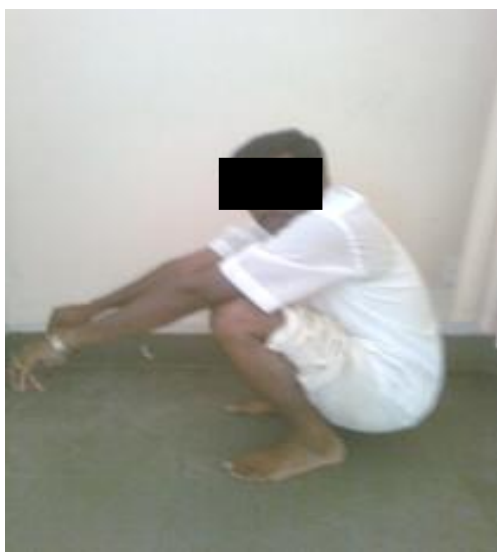

Squatting

Case Series -02

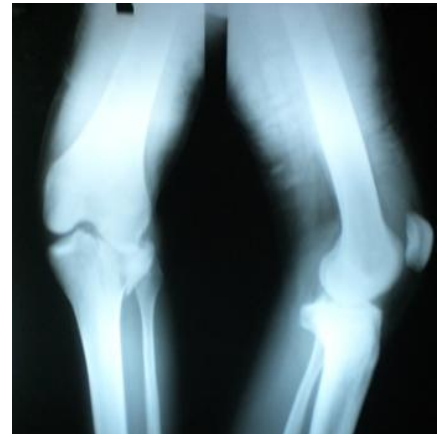

PRE OP Xray

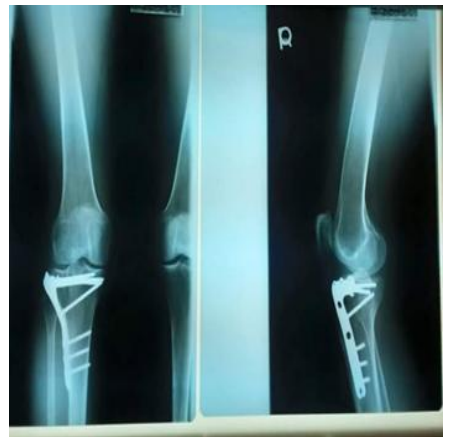

Post OP Xray

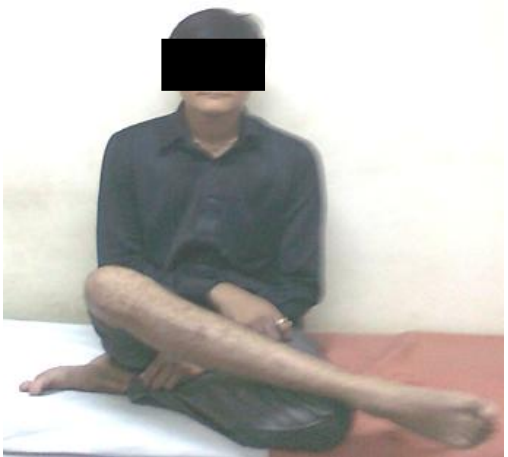

Cross Legged

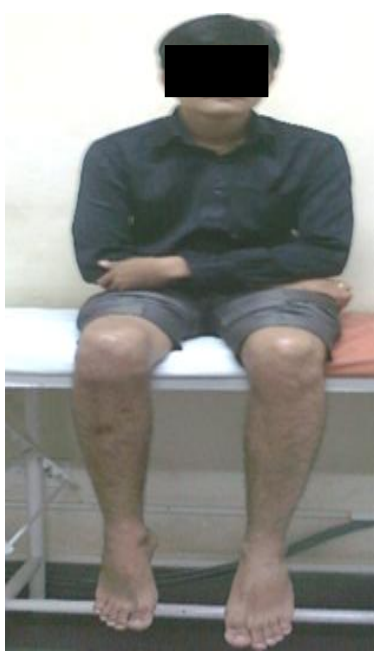

Sitting

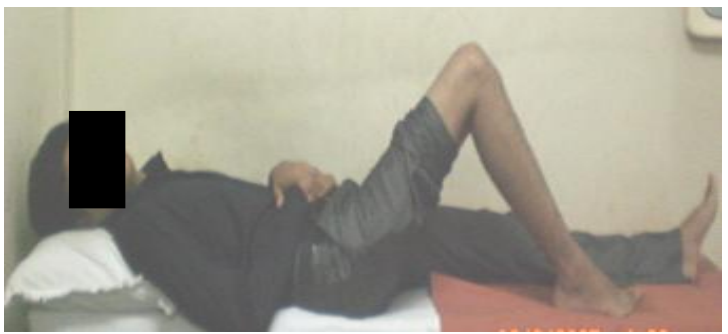

Knee Flexion at $3^{\text {rd }}$ Month

\section{Discussion}

This study comprised of fifty patients with Tibial plateau Fractures who where treated with Locking Compression plate by minimally invasive technique. Overall final outcome was assessed in terms of regaining the lost knee function using NEER'S Score ${ }^{[6]}$.

Out of twenty case series we had 41 closed fractures and 09 open fracture. 38 patients were males and 12 patients were females. The median age was 45 years ranging from 20-70 years. 36 of the fractures were caused by road traffic accidents and 14 were due to fall. 31 patients were with fracture on right side and 19 on left side.

The advent and development of locking compression plate method has effectively improved tibial plateau fractures as common complex fractures ${ }^{[2]}$. The present study shows good results for tibial plateau fractures treated with locking compression plate by minimally invasive technique with regard to functional rating as per Neer's rating score. On the other hand, to improve the knee functional score and minimize postoperative pain, considering locking plate is preferable to nonlocking plate. Although this superiority seems to be preserved in terms of other parameters including 
ROM index, function score, bone-graft need, and even postoperative complications, because of employing small sample size in our study, no statistically significant differences were witnessed. Other studies with similar sample sizes obtained similar success in the use of locking plates.

Stoffel et al ${ }^{[8]}$ collected data from a series of 39 tibial plateau fractures, all of which healed without further intervention and with only two superficial wound infections as complications. Cole et al ${ }^{[9]}$ reported the results of 42 consecutive tibial plateau fractures with $91 \%$ union, $9 \%$ malalignment, and $4 \%$ infection rate. A study by Egol et al ${ }^{[10]}$ reported that 37 of 38 fractures healed without complication and with acceptable alignment employing this treatment method. Most recently, a study by Messmer et al ${ }^{[11]}$ also demonstrated results with no loss of reduction, nonunion, and infection developing in only two of 35 fractures. Krupp et al. found that locked plating was associated with a decreased time to union, decreased incidence of articular malunion, decreased knee stiffness, and decreased overall complications. Moreover, Gossling et al ${ }^{[12]}$ discovered that internal fixation with locking plates, following the principles of minimally invasive percutaneous osteosynthesis, could provide satisfactory fracture reduction with good results regarding the midterm clinical outcome.

Usually a locking plate is inserted providing a raft of proximal locking screws to support the articular surface, buttressing the lateral wall of the proximal tibia and extending distally to adequately support the construct ${ }^{[13]}$. Because the main goal of the treatment is to restore the congruence of the articular surface supporting the tibial plateau cartilage which is usually depressed, to fix the fracture with a stable device, and finally to allow early rehabilitation, the locking plate method can result in achieving main therapeutic goals with appropriate long-term surgical outcome ${ }^{[14]}$.

Common modes of injury in this study were vehicle accidents $(72 \%)$ and fall from height $(28 \%)$. This fracture usually occurs in middle and old age probably due to reduced strength of subarticular calcellous bone. In Rasmussen P.S. (1973) series average age was 55 years. In our study, average age was 45 years. Antero-posterior, lateral and oblique $\mathrm{x}$-rays are sufficient to diagnose the extent of depression, displacement and type of fracture.

In this present study we preferred Schatzker Classification because it is simple, uncomplicated and no special $\mathrm{x}$-rays are required. Its practical utility to carry out treatment and prognosis has got an upper hand. From the observation tables, it is clear that type 2 and 4 fractures are more common followed by type 1,3,5 and 6. According to Muller M.E., Allgower M. and Willengger H. type I is uncommon, but in our study it was $12 \%$. Type 4 fracture is usually found in pure compression type of injuries. Type 2 are commonly found in old persons while type 1 and 4 are commonly found in young patients. Lateral condyle is most commonly affected followed by medical condyle and finally bicondylar. (Hohl M. 1967). In our study lateral condyle fractures were commoner than the medial condyle fracture.

Diagnosis of ligamental injuries should be made at the earliest to prevent residual disabilities. In Rasmussen P.S. series (1973) overall incidence of ligamental injury was $2.5 \%$. According to Schulak D.J. and Gunn D.R. (1975) wedge and bicondylar fractures are commonly associated with ligamental injury. According to Muller M.E. Algower and Willengger depressed type of fractures are very commonly associated with ligamental injury (20\%). In our study incidence of ligamental injury is $10 \%$ (5 Medial Collateral ligaments).

Aim of our study was to achieve anatomical reduction, rigid internal fixation and early mobilization. We have followed minimally invasive Technique under image intensifier. Surgery requires skill, proper technique and instrumentation. Associated ligamental injury was repaired at the time of Surgery. Knee bending was started after 24-48 hours, physiotherapy (CPM) was initiated, while mobilization with crutches was allowed after removal of stitches. Average duration of hospitalization was 12 days.

We had five cases of delayed union. On analyzing it retrospectively we believe the reason for delayed union was due to nature of fracture. 3 case developed post op wound infection, knee pain in 7 cases and stiffness in 12 patients. Interval between injury and time of surgery was within 24 hours for 8 patients, 24 - 48 hours for 21 patients and 48-72 hours for 17 patients and 4 after 72 hours. The reason for this being the younger and middle aged patients with no or minimal comorbidities. Complications like infection that came up in 3 patients was subjected to culture and sensitivity and good antibiotic cover was given accordingly and daily dressing was done until infection subsided. For patients with stiffness again physiotherapy was started with active and passive knee exercises, CPM and wax therapy.

\section{Conclusion}

1. Locking compression plate is a good fixation system for tibial plateau fractures.

2. Operative time is certainly reduced when working with Locking Compression Plate since surgical dissection is kept to a minimum.

3. The device provides good angular stability by its triangular reconstruction principle and thus helps in early mobilization, even in where other modes of fixation often tend to delay the process of mobilization because of lack of stability

4. In contrast to other studies were LCP was used, this present study used the plate through minimally invasive technique. However, when compared with other techniques of plating, in minimally invasive technique the soft tissue damage is considerably less, since periosteal stripping and soft tissue exposure can be kept to a minimum.

5. There was not much difference in individual fracture type healing and weight bearing.

Thus, locking compression plate is an optimal tool for tibial plateau fractures. Minimally invasive technique under the guidance of image intensifier provides better plate placement, significantly less periosteal stripping and soft tissue exposure. However careful understanding of its basic principles, identification of appropriate fracture patterns for use of LCP is essential to avoid complications.

To conclude, Locking Compression Plate is an important armamentarium in treatment of fractures of tibial plateau. However a more comprehensive study with longer follow up periods is essential to throw more light into the advantages, complications and possible disadvantages of the use of Locking Compression Plate with special attention to the long term outcomes.

\section{References}

1. Weigel DP, Marsh JL. High energy fracture of tibial plateau, knee function after longer follow up. JBJS Am. 2002; 84-A(9):1541-51.

2. Kenneth A, Egol, Kennith J. Koval. Fracture of proximal tibia. In: Robert and henchman editors. Roockwood and 
Green's fracture in adulta. $6^{\text {th }}$ edn, Philadelphia, USA. 2005, 1999-2025.

3. Wagner M. General principals for the clinical use of LCP. Injury. 2003; 34(2):B31-42.

4. Testsutaro S, Shimpei H, Toyohiro, Noribumi K, Keiji S. The treatment result of locking compression plate system in proximal tibial fractures. Central Japan Journal of orthopaedic surgery and traumatology. 2005; 48:187.

5. Whittle AP, George W. Wood II. Fracture of proximal tibia (tibial plateau). In: Canale ST, edt., Campbell's operative orthopaedic's, $10^{\text {th }}$ edn. 2005, 2783-2796.

6. Neer CS. Grantham SA, Shelton ML. Supracondylar Fracture of the Adult Femur. J. Bone Joint Surg. 1997; 49A:591-613.

7. Schatzker J. Fractures of the Tibial Plateau. In Schatzker, J., Tile, M. (eds.): Rationale of Operative Fracture Care, New York: Springer, 1987, 279.

8. Stoffel K, Dietaru. Biomechanical testing of the LCPhow can stability in locked internal fixator be controlled. Injury. 2003; 34(2):B11-9.

9. Cole Peter MD, Zlowodzke Micheal MD, Kregor Philip J MD. Treatment of proximal tibial fractures using the Less Invasive Stabilisation System: Surgical Experience and Early Clinical results in 77 fractures. Journal of orthopaedic trauma. 2004; 18(8):528-535.

10. Egol KA et al. Split Depression Tibial Plateau Fractures., J. Orthop. Trauma. 2002; 16:172-7.

11. Messmer P, Gross T. New stabilization technique for fixation of proximal tibia fracture (LISS/LCP).The Umsch. 2003; 60(12):762.

12. Gossling HR, Peterson CA. A New Surgical Approach in the Treatment of Depressed Lateral Condylar Fractures of the Tibia. Clin. Orthop. 2009; 140:96.

13. Stevens DG, Beharry R. The Long-term Functional Outcome of Operatively Treated Tibial Plateau Fractures., J. Orthop. Trauma. 2001; 15:312-20.

14. Stannard JP, Wilson TC, Volgas DA, Alonso JE. The Less Invasive Stabilization System in the Treatment of Complex Fractures of the Tibial Plateau: short-term results. J. Orthop Trauma. 2004; 18(8):528-35. 